\title{
PETERSON'S THEOREM ON SURFACES CORRESPONDING BY PARALLELISM. I
}

\section{G. MARGULIES}

1. Introduction. In a recent series of papers in this journal, Drăgilă $[1 ; 2 ; 3 ; 4 ; 5 ; 6]$ discusses the mapping induced between two real surfaces when these are referred to a common parameter system and such that their normals at corresponding parameter values are parallel. Peterson's theorem in this connection $[7 ; 8, \mathrm{pp} .5,6,26]$ states (roughly) that there then exists a set of corresponding nets in these surfaces such that (a) these nets are parallel, i.e. the field of tangent vectors to the curves of one net is parallel to the corresponding field of tangent vectors to the curves of the other net, and (b) each net is conjugate in the surface in which it lies. Drăgilă $[1$, p. 189] claims that Peterson's theorem is incorrect from two points of view and justifies his claims by constructing examples exhibiting surfaces corresponding by parallel normals for which (1) no such corresponding parallel-conjugate nets exist, and (2) corresponding parallel nets exist but are not conjugate. As far as (1) is concerned, this is indeed the case in the real domain, the reason being (essentially) that the eigenvalues of a real, not necessarily symmetric, linear transformation need not be real. As far as (2) is concerned, it depends on the fact that Drăgilă uses "parallel nets" in a wider sense; he considers two corresponding nets, such that each curve of the first net is parallel not to the corresponding curve of the second net, but to the other curve (if the mapping is given by equal parameters then the $u$-curves of the first net are parallel to the $v$-curves of the second net, and conversely). Drăgilă's results in this direction are therefore not in contradiction to, but rather independent of Peterson's theorem.

It is our purpose to take up and to complete Peterson's theorem, and to put it into a parameter-invariant form by the consideration of the induced mapping of directions (this will be called the mapping projectivity). Specifically, we will show that the parallel-conjugate nets of Peterson are, in each surface, the eigenvector fields of a certain local linear transformation of the surface (considered as Riemann space $V_{2}$ ) into itself, the transformation being generated by a real, not necessarily symmetric, quadratic tensor in $V_{2}$. This coordinatefree characterization of the Peterson nets thus completes the theorem by putting it in a single invariant form valid for both the real and the complex case. Indeed, if the eigenvalues of the linear transformation

Received by the editors July 29, 1960. 
are complex, the nets generated as the trajectories of the corresponding eigenvectors may be considered to exist in the same sense that one considers, for example, the net of asymptotic lines of a real sphere in classical differential geometry. With some modifications, the invariant technique which is used can be adapted to treat analogous problems for higher-dimensional (curved) manifolds embedded in Euclidean space. If the embedding space is not flat, the situation becomes somewhat more complicated because of Levi-Civita parallelism.

2. The mapping of two surfaces in $E_{3}$. Let $r^{i}\left(u^{1}, u^{2}\right)$ and $R^{i}\left(u^{1}, u^{2}\right)$ be radius vectors of two real surfaces $(\mathfrak{r})$ and $(\Re)$ in $E_{3}$, and assume these functions are of class $C^{m}, m \geqq 3$. A given parameter point $(u)$ $=\left(u^{1}, u^{2}\right)$ determines a point in each surface and thus induces a mapping of one onto the other. At regular surface points, this mapping may be described [9] by the set of partial differential equations

$$
R_{\lambda}^{i}=s_{\lambda}^{\alpha}(u) r_{\alpha}^{i}+z_{\lambda}(u) n^{i}
$$

where, letting $\partial_{\lambda}=\partial / \partial u^{\lambda}$, we have set $R_{\lambda}^{i}=\partial_{\lambda} R^{i}, r_{\alpha}^{i}=\partial_{\alpha} r^{i}$, and $n^{i}$ is the unit normal to the surface (r). (Latin indices have the range 1, 2, 3 and pertain to vectors in $E_{3}$, while Greek indices have the range 1, 2 and denote tensor components in the surfaces $(\mathfrak{r})$ and $(\Re)$ considered as Riemann spaces $V_{2}$. The summation convention is used throughout.)

Let $g_{\alpha \beta}$ be the metric tensor of the surface $(\mathfrak{r})$. Then from (2.1) we have ${ }^{1}$

$$
\delta_{i j} r^{i}{ }_{\mu} R_{\lambda}^{i}={ }_{s_{\lambda} \delta_{i j} r^{i}{ }_{\alpha} r^{i}{ }_{\mu}=s^{\alpha}{ }_{\lambda} g_{\alpha \mu}=s_{\mu \lambda}}
$$

and we call $s_{\mu \lambda}$ the mapping tensor. Let $\nabla_{\lambda}$ be the covariant derivative w.r.t. $g_{\alpha \beta}$, and $h_{\alpha \beta}$ the second fundamental tensor of the surface $(\mathfrak{r})$. Then from (2.1) one obtains

$$
\partial_{\mu} R_{\lambda}^{i}=\left\{\nabla_{\mu} s_{\lambda}^{\alpha}-h_{\mu}^{\alpha} z_{\lambda}+\Gamma_{\lambda \mu}^{\beta} s_{\beta}^{\alpha}\right\} r_{\alpha}^{i}+\left\{s^{\alpha} h_{\alpha \mu}+\nabla_{\mu} z_{\lambda}+\Gamma_{\mu \lambda}^{\beta} z_{\beta}\right\} n^{i}
$$

where $\Gamma_{\lambda \mu}^{\beta}$ denotes the Christoffel symbols (of the second kind) of the surface $(\mathfrak{r})$. Taking the skew-symmetric part of (2.3), it follows then that

${ }^{1}$ We assume, for the sake of simplicity, that a rectangular cartesian system is used in $E_{3}$. One could just as easily use an affine system, with metric tensor $a_{i j}$, in which case one would have $s_{\lambda \mu}=a_{i j} r^{i} R^{i}{ }_{\mu}$, all other results following with a similar modification. 


$$
\begin{aligned}
\nabla_{[\mu} s_{\lambda]}^{\alpha}-h_{\left[\mu z_{\lambda]}\right.}^{\alpha} & =0, \\
\left.\nabla_{\left[\mu z_{\lambda]}\right.}+h_{\alpha[\mu} s^{\alpha}\right] & =0
\end{aligned}
$$

are the integrability conditions for the differential equations (2.1). It can be shown (see [9]) that if only (r) and $s_{\alpha \beta}, z_{\beta}$ are given, then these integrability conditions (together with a regularity condition) are n.a.s.c.'s for the existence of $(\Re)$, determined then up to a translation w.r.t. (r) in $E_{3}$. If both surfaces are given, these conditions must be satisfied. In case of parallel normals (parallel tangent planes) we obviously have $z_{\lambda}=0$, and the above conditions reduce to

$$
\begin{aligned}
\nabla_{[\mu} s_{\lambda]}^{\alpha} & =0, \\
h_{\alpha[\mu} s_{\lambda]}^{\alpha} & =0 .
\end{aligned}
$$

This shows, among other things, that the mapping tensor $s^{\alpha}{ }_{\beta}$ cannot be chosen arbitrarily to define a mapping.

3 . The two projectivities in $V_{2}$. There is a naturally induced projectivity between the tangent planes of two surfaces mapped upon each other by means of equal parameters. Since $d u^{\lambda}=d u^{\lambda}$, the vector $d r^{i}=d u^{\lambda} r_{\lambda}{ }_{\lambda}$ is projective with the vector $d R^{i}=d u^{\lambda} R_{\lambda}^{i}$. Thus two vector fields $v^{i}=v^{\lambda} r_{\lambda}^{i}$ in $(\mathfrak{r})$ and $V^{i}=V^{\lambda} R_{\lambda}^{i}$ in $(\Re)$ will be called projective if and only if $V^{\lambda}=v^{\lambda}$. We shall refer to this projectivity as the mapping projectivity.

There is a second projectivity which, under the mapping (2.1), takes the tangent plane of $(\mathfrak{r})$ into itself. Let the fields $v^{i}$ and $V^{i}$ be projective under the mapping projectivity, and let $*_{v^{i}}{ }^{*} *_{v^{\lambda}} r_{\lambda}$ be the orthogonal projection of $V^{i}$ on the tangent plane of (r) (see Figure 1). Then from (2.1) we have $V^{i}=V^{\lambda} R_{\lambda}^{i}=V^{\lambda}\left(s^{\alpha}{ }_{\lambda} r_{\alpha}{ }_{\alpha}+z_{\lambda} n^{i}\right)$. Since $v^{i}$ is projective with $V^{i}$, we have $v^{\lambda}=V^{\lambda}$, hence $V^{i}=v^{\lambda}\left(s^{\alpha}{ }_{\lambda} r_{\alpha}{ }^{2}+z_{\lambda} n^{i}\right)$, and the required orthogonal projection $*^{i}$ of $V^{i}$ is then determined by

$$
*_{v}{ }^{\alpha}=s_{\beta^{v}}^{\alpha} .
$$

The linear transformation between vector fields of $(\mathfrak{r})$ described above by (3.1) will be called the local projectivity. It follows trivially that if the mapping is a similarity transformation, i.e. if $z_{\lambda}=0$ and $s_{\mu}^{\lambda}=\psi \delta_{\mu}^{\lambda}$ for some absolute scalar field $\psi(u)$, then the local projectivity reduces to the quadratic involution $s^{\alpha}{ }_{\lambda} s_{\beta}=\psi^{2} \delta_{\beta}^{\alpha}$. While the local projectivity is a linear transformation which takes a $V_{2}$ locally into itself, the mapping projectivity is a linear transformation between two $V_{2}$ 's with different metrics. It can be shown [9] that the covariant form of the condition $V^{\lambda}=v^{\lambda}$ is given by $V_{\mu}=g^{\lambda \omega}\left(s_{\alpha \lambda} s^{\alpha}{ }_{\mu}+z_{\lambda} z_{\mu}\right) v_{\omega}$, where $g^{\lambda \omega}$ is inverse to $g_{\omega \mu}$. 


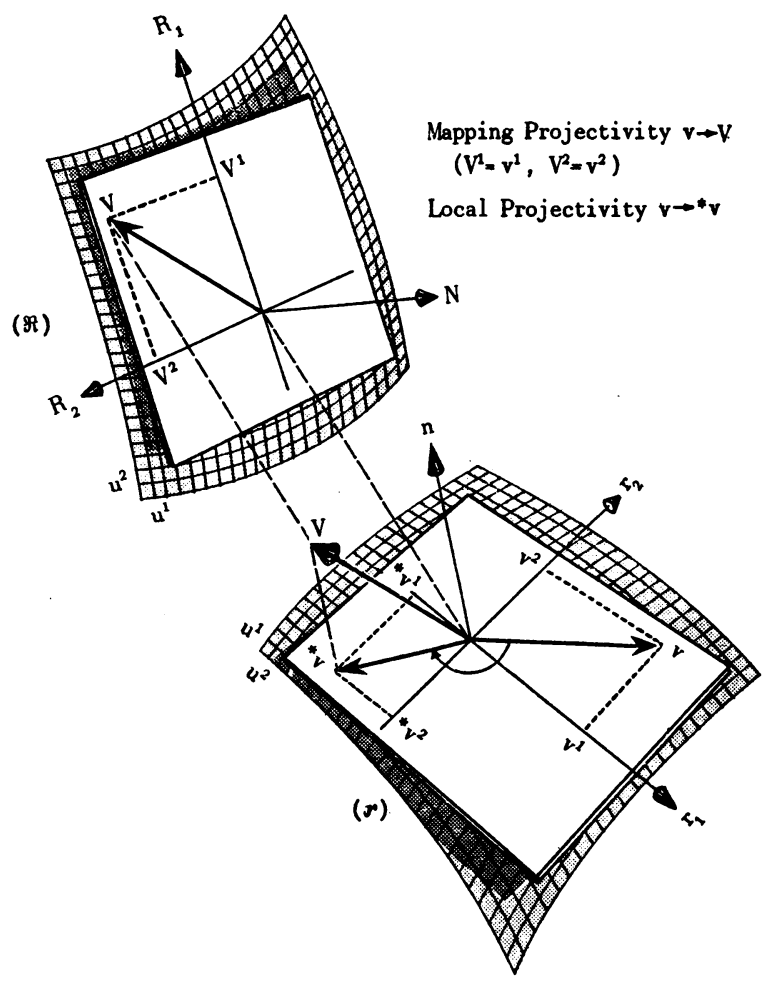

FIGURE 1

4. Peterson nets. We shall call trajectories of a vector field the system of curves whose tangent vectors are the field vectors. Referring now to the mapping projectivity $(\S 3)$ we give

Definition 4.1. By the Peterson nets of the pair of surfaces $(\mathfrak{r}),(\Re)$ we mean the trajectories of a pair of distinct conjugate vector fields $\left(p^{\lambda}, q^{\lambda}\right)$ in (r) together with the trajectories of the projectively corresponding fields $\left(P^{\lambda}=p^{\lambda}, Q^{\lambda}=q^{\lambda}\right)$ in $(\Re)$, the fields $\left(P^{\lambda}, Q^{\lambda}\right)$ being required to be also conjugate in $(\Re)$. (These nets were named "courbes de base" by Peterson $[8$, pp. 5, 6, 26] for the special case where the surfaces have parallel tangent planes at corresponding points. These nets have then the further property of being parallel, as described above in $\$ 1$. We shall deal with this case below in §5.) If the mapping projectivity maps asymptotic lines in (r) upon asymptotic lines in $(\Re)$, then we shall say in brief that the mapping preserves asymptotic directions (hence preserves also all conjugate systems). Definition 4.1 is not void in view of 
TheOREM 4.1. There always exists at least one set of Peterson nets. If the mapping does not preserve asymptotic directions, the Peterson nets are unique.

Proof. A conjugate net $\left(p^{\lambda}, q^{\lambda}\right)$ in $(\mathfrak{r})$ corresponds under the mapping projectivity to a net $\left(p^{\lambda}, q^{\lambda}\right)$ in $(\Re)$. If this net is to be also conjugate in $(\Re)$, then the following two conditions must hold (we designate the second fundamental tensor of $(\Re)$ by $\left.H_{\lambda_{\mu}}\right)$ :

$$
\begin{aligned}
h_{\lambda \mu} p^{\lambda} q^{\mu} & =0, \\
H_{\lambda \mu} p^{\lambda} q^{\mu} & =0 .
\end{aligned}
$$

This amounts then to determining fields $p^{\lambda}, q^{\lambda}$ conjugate w.r.t. two tensors $h_{\lambda \mu}$ and $H_{\lambda \mu}$. It is well known (e.g. see [10, pp. 145-146]) that such fields always exist, being determined up to a multiplicative factor from the roots of the quadratic equation $e^{\alpha \beta} H_{\lambda \alpha} h_{\mu \beta} x^{\lambda} x^{\mu}=0$, where $e^{\alpha \beta}$ is the contravariant permutation tensor in the surface (r). Furthermore, if the tensors $h_{\lambda \mu}, H_{\lambda \mu}$ are not proportional (equivalently: if the mapping does not preserve asymptotic directions), then these fields are unique.

5. Surfaces corresponding by parallelism. We turn now to the class of surface mappings considered by Peterson $[7 ; 8]$. Here the normals at corresponding points in each surface are parallel. We call these mappings by parallelism. For such mappings, it is now possible to obtain an important relation ${ }^{2}$ between the mapping tensor $s^{\lambda}{ }_{\mu}$ and the tensors $h_{\lambda_{\mu}}, H_{\lambda_{\mu}}$, and, at nonparabolic points, an explicit expression for $s_{\lambda \mu}$ in terms of $h_{\lambda \mu}, H_{\lambda \mu}$ and their inverses ${ }^{-1} h_{\lambda \mu},{ }^{-1} H_{\lambda \mu}$ :

Lemma 5.1. For mappings by parallelism we have

$$
H_{\omega \mu}=s^{\alpha}{ }_{\omega} h_{\mu \alpha}
$$

and at nonparabolic points ${ }^{3}$

$$
s_{\lambda \omega}={ }^{-1} h_{\lambda}^{\beta} H_{\omega \beta}=h_{\lambda \alpha}{ }^{-1} H_{\omega}^{\alpha} .
$$

Proof. Let $n^{i}, N^{i}$ be the respective unit normals of $(\mathfrak{r})$ and $(\Re)$. Starting with the mapping equations $R^{i}{ }_{\mu}=s^{\alpha}{ }_{\mu} r_{\alpha}{ }_{\alpha}$, we take a scalar

2 This is also possible for general mappings, but the resulting formulas are considerably more complicated (see [9, Theorem 3.4, p. 172]).

3 Tensors in $(\Re)$, with upper-case kernel letters, have their indices lowered and raised by $G_{\alpha \beta}, G^{\alpha \beta}$. Tensors in (r), with lower-case kernel letters, have their indices lowered and raised by $g_{\alpha \beta}, g^{\alpha \beta}$. From (2.1) it follows that $G_{\lambda \mu}$ and $g_{\lambda \mu}$ are related by $G_{\lambda \mu}=s^{\alpha} \lambda^{\beta}{ }^{\beta}{ }_{\mu}+\Sigma_{\lambda} z_{\mu}$ for a general mapping. ( $G_{\lambda \mu}$ denotes the metric tensor of the surface $(\Re)$, and $G^{\lambda \mu}$ denotes its inverse.) 
product on the left with $\partial_{\omega} N^{i}$, on the right with $\partial_{\omega} n^{i}$, since $n^{i}=N^{i}$. Using then Weingarten's equations of surface theory yields (5.1). Multiplying through, at nonparabolic points, by ${ }^{-1} h_{\lambda}^{\mu}$ then gives ${ }^{-1} h_{\lambda}^{\mu} H_{\omega \mu}=s^{\alpha}{ }_{\omega} h_{\mu \alpha}{ }^{-1} h_{\lambda}^{\mu}=s^{\alpha}{ }_{\omega} g_{\alpha \lambda}=s_{\lambda \omega}$, which is the first equality in (5.2). For the second equality, observe that the metric tensors of the spherical images of $(\mathfrak{r})$ and $(\Re)$ must necessarily be identical, i.e. ${ }^{(8)} h_{\alpha \beta}={ }^{(s)} H_{\alpha \beta}$, or explicitly $h_{\alpha}^{\mu} h_{\mu \beta}=H_{\alpha}^{\nu} H_{\nu \beta}$. Multiplying through, at nonparabolic points, by ${ }^{-1} h_{\lambda}^{\beta-1} H_{\omega}^{\alpha}$ then yields the desired result.

REMARK. It is clear from (5.1) that integrability condition (2.7) is equivalent to $H_{[\omega \mu]}=0$, i.e. to the symmetry of $H_{\omega \mu}$. The verification of integrability condition (2.6) will involve the Codazzi-Mainardi equations, as can be seen by the position of the operator $\nabla_{\mu}$ in (2.6). However, because the second fundamental tensors $h_{\lambda \mu}$ and $H_{\lambda \mu}$ of the two surfaces are involved, it is necessary to introduce the relationship between the Christoffel symbols of the two surfaces (see [9, Theorem 3.3 , p. 172]).

Let the scalar densities $\boldsymbol{g}, \boldsymbol{G}, \boldsymbol{h}, \boldsymbol{H}$ denote the respective determinants of the tensors $g_{\alpha \beta}, G_{\alpha \beta}, h_{\alpha \beta}, H_{\alpha \beta}$, and the absolute scalars $s, k, K$ denote respectively the determinant of $s^{\alpha}{ }_{\beta}$ and the Gauss curvatures of the surfaces $(\mathfrak{r})$ and $(\Re)$.

Lemma 5.2. For mappings by parallelism $s=(G / g)^{1 / 2} \cdot{ }^{4}$ Furthermore, parabolic points in one surface correspond to parabolic points in the other surface. At nonparabolic points, the ratio of the Gauss curvatures is $k / K=s$.

Proof. From the mapping equations (2.1) we may compute the unit normal of the surface $(\Re)$ for the case of a general mapping and obtain (see [9, Theorem 3.2, p. 171])

$$
N^{i}=(g / G)^{1 / 2}\left\{s n^{i}-e^{\alpha \beta} e^{\omega \lambda} s_{\beta \lambda} z_{\omega} r^{i}{ }_{\alpha}\right\}
$$

where $e^{\alpha \beta}$ is the contravariant permutation tensor in the surface $(\mathfrak{r})$. For mappings by parallelism $z_{\lambda}=0, n^{i}=N^{i}$ and the result $s=(G / \boldsymbol{g})^{1 / 2}$ follows. It is clear from the above expression for the normal that $s=0$ if and only if the tangent planes of the two surfaces are mutually orthogonal at corresponding points, which is impossible for a mapping by parallelism. Thus $s \neq 0$, and taking determinants in (5.1) yields $H=s h$, which shows that parabolic points must be in correspondence (a result which is also evident by a purely geometric argument in $E_{3}$ ). At nonparabolic points, we take determinants in

${ }^{4}$ If $n^{i}=N^{i}$, we choose the positive root. If $n^{i}=-N^{i}$, the negative root must be taken. It is interesting to compare this result with E. K. Hsiao's [12, Theorem 1, p. 355]. 
the second equation of (5.2), rewriting it however first as $g_{\alpha \lambda} s^{\alpha}{ }_{\omega}$ $=h_{\lambda \alpha}{ }^{-1} H_{\beta \omega} G^{\alpha \beta}$. This yields $(\boldsymbol{H} / G) s=h / \xi$, hence $k / K=s$, which is the desired result. This completes the proof.

REMARK. Similarly, of course, elliptic points and hyperbolic points are also (respectively) in correspondence between the two surfaces. For anti-parallel mappings, i.e. $n^{i}=-N^{i}$, it follows then that elliptic points are mapped upon hyperbolic points, and vice-versa.

Mappings which preserve asymptotic directions appeared previously in Theorem 4.1, and we may now characterize these for the case when these mappings are also by parallelism. They turn out to be trivial, i.e. a change of scale and translation in $E_{3}$. From the point of view of mappings by parallelism, such mappings may (but need not) be excluded.

Lemma 5.3. A mapping by parallelism which preserves asymptotic directions is the product of a homothetic transformation and a translation in $E_{3}$.

Proof. From (5.1) it is clear that if the mapping is a similarity transformation, i.e. $s_{\omega}^{\lambda}=\psi \delta_{\omega}^{\lambda}$, then $H_{\lambda \mu}$ and $h_{\lambda \mu}$ are proportional and asymptotic directions are preserved. (The converse is trivial.) To show that $\psi=$ const., we use the integrability conditions (2.6) which yield $\nabla_{[\mu} \psi \delta_{\lambda]}^{\alpha}=0$, and since $\psi$ is an absolute scalar field, the result follows. Thus the mapping reduces to $R_{\lambda}^{i}=\psi r_{\lambda}^{i}$, an integral of which is $R^{i}=\psi r^{i}+$ const. (Observe that the other integrability condition, i.e. $h_{\alpha[\mu} \psi \delta_{\lambda]}^{\alpha}=\psi h_{[\lambda \mu]}=0$ is trivially satisfied.)

In considering a nonsymmetric quadratic tensor, e.g. $s_{\lambda \mu}$, there are two types of eigenvectors (one for each index, so to speak). We shall restrict our attention to one type only and call $v^{\lambda}$ an eigenvector of $s^{\lambda}{ }_{\mu}$ if for some absolute scalar $\theta$ we have $\theta v^{\lambda}=s^{\lambda}{ }_{\omega} v^{\omega}$. Also, in dealing with corresponding nets, we shall call them parallel if corresponding curves have parallel tangent vectors at corresponding points. Before stating Peterson's theorem, we observe that the mapping projectivity, by "equal contravariant components," is obviously unchanged if we interchange the roles of the two surfaces. The local projectivity, however, is a linear transformation which takes one of the surfaces (locally) into itself and is determined by the mapping tensor $s_{\mu}{ }_{\mu}$. If we wish to interchange the role of the two surfaces, we may introduce, for surfaces corresponding by parallelism, the inverse mapping $r^{i}{ }_{\lambda}=S^{\alpha}{ }_{\lambda} R_{\alpha}^{i}$, and call $S^{\alpha}{ }_{\lambda}$ the inverse-mapping tensor. It is then obvious that $S^{\alpha}{ }_{\lambda}$ is inverse to the mapping tensor $s^{\lambda}{ }_{\mu}$, i.e. $S^{\alpha}{ }_{\lambda} s_{\beta}{ }_{\beta}=\delta_{\beta}^{\alpha}, s^{\alpha}{ }_{\lambda} S_{\beta}{ }_{\beta}$ $=\delta_{\beta}^{\alpha}$, and in fact $S_{\lambda \mu}=s_{\mu \lambda} .{ }^{5} \mathrm{We}$ may thus speak of the local projectiv-

\footnotetext{
5 See footnote 3. For properties of the inverse-mapping tensor for a general mapping, see $[9, \S 5]$.
} 
ity in each surface of the mapping. Furthermore, it is clear that the eigenvalues of one local projectivity are the reciprocals of the eigenvalues of the other local projectivity, and that the corresponding eigenvectors are projective under the mapping projectivity. We are now in position to state in invariant (i.e. coordinate-free) manner the complete form of Peterson's theorem for mappings by parallelism:

THEOREM 5.1. If two surfaces in $E_{3}$ correspond by parallelism in such a way that their local projectivities have distinct eigenvalues, then their Peterson nets are, in each surface, the trajectories of the eigenvector fields of the local projectivity. Furthermore, these nets are parallel.

Proof. In view of the remarks preceding the theorem, it suffices to restrict our attention to one of the surfaces only, say $(\mathfrak{r})$. In that surface, the local projectivity is determined by the mapping tensor $s^{\lambda}{ }_{\mu}$. Let $p^{\lambda}$ and $q^{\lambda}$ be two eigenvectors corresponding to the two distinct eigenvalues $\theta_{p}$ and $\theta_{q}$ of $s^{\lambda}{ }_{\mu}{ }^{6}$ Then:

$$
\begin{aligned}
& \theta_{p} p^{\lambda}=s^{\lambda}{ }_{\omega} p^{\omega}, \\
& \theta_{q} q^{\lambda}=s^{\lambda}{ }_{\omega}^{\omega},
\end{aligned}
$$

and using (5.1) we obtain, after multiplying through by $h_{\lambda_{\mu}}$ :

$$
\begin{aligned}
\theta_{p} p^{\lambda} h_{\lambda \mu} & =p^{\omega} H_{\omega \mu}, \\
\theta_{q} q^{\lambda} h_{\lambda \mu} & =q^{\omega} H_{\omega \mu} .
\end{aligned}
$$

It then follows that $\left(\theta_{p}-\theta_{q}\right) h_{\lambda \mu} p^{\lambda} q^{\mu}=0$, and since $\theta_{p} \neq \theta_{q}$, we have

$$
h_{\lambda \mu} p^{\lambda} q^{\mu}=0 \text {. }
$$

Multiplying the first equation in (5.4) by $q^{\mu}$ and using (5.5) then yields

$$
H_{\omega \mu} p^{\omega} q^{\mu}=0 .
$$

Thus the eigenvector fields of $s^{\lambda}$ must be conjugate w.r.t. both tensors $h_{\lambda \mu}$ and $H_{\lambda \mu}$. By Theorem 4.1, their trajectories determine thus the Peterson nets. To prove the parallelism of these nets, observe that for the eigenvalue $\theta$ and the corresponding eigenvector $v^{\lambda}$, we have $v^{\lambda} R_{\lambda}^{i}=v^{\lambda}{ }^{\omega}{ }_{\lambda} r^{i}{ }_{\omega}=\theta v^{\omega} r^{i}{ }_{\omega}$, hence the eigenvector fields of the local projectivity generate parallel directions in (the tangent planes of) the two surfaces. This completes the proof.

6. Conclusion. It is now clear that if one includes eigenvectors corresponding to complex(-conjugate) eigenvalues of the local pr jiectiv-

- The latin indices $p, q$ are nontensorial here. Also, these eigenvalues need not be real. 
ity, Peterson's theorem is valid. This clarifies then the status of Drăgilă's first counter-example [1, p. 191]. His second one corresponds, in our notation, to the case where the mapping tensor $s^{\lambda}{ }_{\mu}$ is chosen to be

$$
\left(\begin{array}{ll}
s^{1}{ }_{1} s^{2}{ }_{1} \\
s^{1}{ }_{2} & s^{2}{ }_{2}
\end{array}\right)=\left(\begin{array}{ll}
0 & \lambda \\
\mu & 0
\end{array}\right) \text {. }
$$

While Drăgilă claims $[1$, p. 193] that $\lambda$ and $\mu$ may be arbitrary functions, they must nevertheless satisfy the integrability conditions (2.6) and (2.7). The latter condition yields $\mu h_{11}=\lambda h_{22}$ and together with the relations derived in (5.1), i.e. $H_{\lambda \mu}=s^{\omega}{ }_{\lambda} h_{\mu \omega}$, one may easily verify that

$$
h_{11} H_{22}+h_{22} H_{11}-2 h_{12} H_{12}=0 .
$$

It is well known (it appears as a problem in Eisenhart's book [11, p. 243, \#12]) that this last equation characterizes (general) mappings for which asymptotic directions in one surface are mapped upon a conjugate system in the other and vice-versa. As far as Peterson's theorem is concerned, this example of Drăgilă does not provide a counter-example because it does not apply. Even though, in this example, the $u$-lines in one surface are parallel to the $v$-lines in the other surface, and conversely, it is unwarranted to assume that these parameter nets should be the Peterson nets. In fact, the parallelism is constructed in this case between the trajectories of the fields $\delta_{1}^{\lambda}$ in one surface and $\delta_{2}^{\lambda}$ in the other; these fields are not projective under the mapping projectivity, and hence Peterson's theorem does not apply. The Peterson nets are, in this case, still determined by the eigenvectors of $s_{\mu}^{\lambda}$. (Whether or not the Peterson nets are real depends here on whether $\lambda$ and $\mu$ are of equal or opposite sign.)

One may observe that if the mapping does preserve asymptotic directions, the Peterson nets are not unique (Theorem 4.1). But since in that case the mapping (if by parallelism) is a similarity transformation: $s_{\mu}^{\lambda}=\psi \delta_{\mu}^{\lambda}$, the eigenvectors of the local projectivity are not unique either-in fact every vector is an eigenvector and hence any conjugate net is a Peterson net. There is thus no need to exclude similarity transformations. It is interesting to observe that if the mapping tensor is symmetric (but not proportional to the metric tensor $\left.g_{\lambda \mu}\right)^{7}$ then the Peterson nets must necessarily be the lines of curvature in the two surfaces.

REMARK. Let the mapping be described in $E_{3}$ by the rectilinear congruence

${ }^{7}$ I.e., if the mapping is not a similarity transformation. 


$$
L^{i}=R^{i}-r^{i}=v^{\lambda}(u) r_{\lambda}^{i}+w(u) n^{i} .
$$

Then from (2.1) we get $s_{\lambda \mu}=g_{\lambda \mu}-w h_{\lambda \mu}+\nabla_{\mu} v_{\lambda}$ and $z_{\mu}=\nabla_{\mu} w-h_{\mu}^{\lambda} v_{\lambda}$. Thus a n.a.s.c. for the mapping tensor to be symmetric is that $v_{\lambda}$ be a gradient field. Eliminating the function $w(u)$ between the two expressions above, one can obtain the differential equations for the congruence of curves $f(u)=$ const. in $(\mathfrak{r})$ for which $v_{\lambda}(u)$ is the gradient field. These are given by

$$
\left[\Delta_{2}(f)+2-s_{\alpha}^{\alpha}\right] \partial_{\lambda}(\log h)-h h_{\lambda}^{\alpha} \nabla_{\alpha} f-\partial_{\lambda} \Delta_{2}(f)=0,
$$

where $\Delta_{2}$ denotes the second Beltrami operator and $h$ the mean curvature of the surface $(\mathfrak{r})$. (For the case where $R^{i}-r^{i}$ forms a parallel rectilinear congruence in $E_{3}$, a geometric interpretation can be given; see $[9$, p. 175].)

What is less clear, at present, is the significance of the requirement that the local projectivity have distinct eigenvalues. Excluding similarity transformations (which represent a trivial case) the case of one eigenvalue of multiplicity 2 with a single corresponding eigenvector leads to a degenerate form of Peterson's theorem: the conjugate nets degenerate into a single asymptotic line. If, furthermore, the mapping is also equiareal, then both surfaces $(\mathfrak{r})$ and $(\Re)$ must be ruled. While we plan to discuss this more at length in the second part of this paper, we conclude with the observation that the existence of the Peterson nets is an invariant property of the mapping of two surfaces in the sense that it does not depend upon the possibility of choosing a special parameter system for the surfaces, as Drăgilă and Peterson also seem to have thought. While the embedding of the surfaces in $E_{3}$ is related (via the normals) to the tensors $h_{\lambda \mu}, H_{\lambda \mu}$, these tensors together with all the other ones are objects in $V_{2}$ and Peterson's theorem thus appears to be a proposition in $V_{2}$ dealing primarily with the purely algebraic properties of the mapping tensor.

\section{REFERENCES}

1. Pavel Drăgilă, Sur la correspondance par parallélisme de deux surfaces, Proc. Amer. Math. Soc. vol. 9 (1958) pp. 189-200.

2. - Correspondances singulières par parallelisme des plans tangents de deux surfaces, Proc. Amer. Math. Soc. vol. 9 (1958) pp. 518-521.

3. - Correspondance entre deux surfaces par trois couples de tangentes parallèles, Proc. Amer. Math. Soc. vol. 9 (1958) pp. 851-854.

4. - Correspondance entre deux surfaces par quatre couples de tangentes parallèles, Proc. Amer. Math. Soc. vol. 9 (1958) pp. 855-859.

5. - Correspondance entre deux surfaces par des faisceaux de tangentes paralleles, Proc. Amer. Math. Soc. vol. 10 (1959) pp. 366-368. 
6. - Sur un système de couples de réseaux parallèles, Proc. Amer. Math. Soc. vol. 11 (1960) pp. 255-264.

7. K. M. Peterson, Ob otnochenia $i$ srodsvah mejdou crivemi povernostemi, Mat. Sb. (1866).

8. - Sur les relations et les affinites entre les surfaces courbes, Ann. Fac. Sci. Univ. Toulouse (1905).

9. G. Margulies, Intrinsic characterization of surface mappings, Tensor (Japan) vol. 10 (1960) pp. 167-188.

10. Václav Hlavatý, Differentialgeometrie und Tensorrechnung, Groningen, P. Noordhoff, 1939.

11. L. P. Eisenhart, An introduction to differential geometry, Princeton, Princeton Univ., 1947.

12. E. K. Hsiao, On the theory of Löbell on transformation of surfaces, Amer. Math. Monthly vol. 67 (1960), pp. 353-356.

Florida State University 